\title{
Hilfestellungen für Gebärende mit PDA
}

\author{
Andrea Mora, Hebamme, Stuttgart
}

\section{In den letzten Jahren stieg die Anzahl der Periduralanästhesien zur Geburtserleichte- rung in Deutschland auf knapp $24 \%$ an. Bei fast jeder 4. Geburt müssen sich die Frau und ihre Hebamme also auch auf die PDA-bedingten Veränderungen einstellen. Dieser Beitrag zeigt neue Möglichkeiten, wie ungünstige Auswirkungen einer PDA auf
den Geburtsverlauf besser kompensiert werden können.}

Die hier vorgestellten Ideen entspringen der praktischen Auseinandersetzung mit dem Bewegungskonzept der Kinästhetik, speziell der MH Kinaesthetics, benannt nach den Begründern Dr. Lenny Maietta und Dr. Frank Hatch. Hier geht es darum, die eigene Bewegung unter verschiedenen Aspekten zu erfahren, Gesetzmäßigkeiten zu entdecken und das integrierte Bewegungsverständnis auf den fachlichen Kontext zu übertragen.

\section{Problem 1: Veränderte Wahrnehmung der Beine}

\section{Die typische Situation}

Oft zeigt sich schon unmittelbar nach dem Legen der PDA (z.B. beim Hochrutschen im Bett oder beim Drehen auf eine Seite), dass die alltäglichen und intuitiven Bewegungen der Gebärenden verändert sind.

Der Grund: Frauen mit PDA nehmen ihre Beine anders (und sehr häufig weniger) wahr. Dies führt zu einer deutlichen Bewegungseinschränkung des Beckens und zu einer erheblichen Störung aller „normalen“ Bewegungsabläufe wie Positionswechsel im Bett - mit dementsprechend großen Einschränkungen bei allen geburtsrelevanten Bewegungen.
Das Ausmaß der motorischen Einschränkung liegt hauptsächlich an folgenden Faktoren:

- Mischungsverhältnis zwischen Lokalanästhetikum und Opiat: Je höher die Dosierung des Lokalanästhetikums, desto größer ist die zu erwartende motorische Blockade. Da das Opiat über den periduralen Venenkomplex teilweise resorbiert wird und damit auf das Kind übergeht, wird die Frage nach dem optimalen Dosierungsverhältnis weiterhin kontrovers diskutiert.

- Position der Frau während der Ausbreitung: Die tägliche Praxis zeigt, dass bei einer einseitig unzureichenden Wirkung der PDA eine Lagerung der Frau auf diese Seite unmittelbar nach der Injektion der Wirkdosis oft eine zügige, seitengleiche Ausbreitung bewirken kann.

Deshalb sollte die Frau nur so lange auf einer Seite oder flach liegen, bis die gewünschte Wirkung erreicht ist.

\footnotetext{
- Dosierung der weiteren Medikamentengaben: Über die Laufrate des Perfusors bei einer kontinuierlichen PDA bzw. über die Höhe und Häufigkeit des verabreichten Mittels bei einer Bolus-PDA können unerwünschte Bewegungseinschränkungen vermindert werden.
}

Obwohl die Anlage und Betreuung der PDA eine ärztliche Aufgabe ist, gehört zu einer optimalen Durchführung der interdisziplinäre Austausch. Hier kann die Frage, was das Ziel der PDA sein soll, ein wichtiger Aspekt sein, die hausinterne Routine zu überdenken. Würde man dazu die Frauen, das geburtshilfliche Team und die Anästhesisten befragen, bekäme man wahrscheinlich sehr unterschiedliche Antworten. Während für die Anästhesie in der Regel eine möglichst komplette Schmerzausschaltung oberste Priorität hat, wünschen sich Hebammen und Geburtshelfer gerade zum Ende der Geburt vor allem eine möglichst geringe motorische Einschränkung.

Die Frauen wünschen sich vor allem eine spontane Geburt und sind in der Regel dankbar, wenn sie die Geburtsphase aktiv gestalten können. Sie würden sich wahrscheinlich immer für eine reduzierte PDA entscheiden, wenn dadurch eine vaginal-operative Geburt verhindert werden kann. Hier liegt es in unserer Verantwortung, die Frauen auch während der Geburt individuell zu beraten, sie (eventuell nach einer wichtigen Pause zum Kräftesammeln) wieder aktiver in die Geburtsarbeit zu bringen und $\mathrm{zu}$ motivieren, sich tatkräftig auf die Austreibungsphase und ggf. auf wiederkommende Schmerzen einzulassen. 


\section{Kinästhetische Analyse}

Im kinästhetischen Konzept „Funktionale Anatomie" wird das Zusammenspiel zwischen Körperstamm und Extremitäten analysiert: Da unsere Extremitäten wesentlich leichter sind als unser Körperstamm, initiieren wir jeden Positionswechsel über unsere Extremitäten. So bewegen wir unser Becken in aller Regel über unsere Beine.

Dieses instinktiv fest verankerte Bewegungsmuster wird bei einer Frau mit liegender PDA zunächst behindert. Denn selbst Frauen, die später mit der PDA umher gehen und sich wieder gut selbstständig bewegen können, bewegen sich zunächst (vor allem so lange die Testdosis noch wirkt) vorsichtiger und deutlich langsamer. Sie greifen oft zum Tuch oder zu einer unterstützenden Person, wenn sie sich hinsetzen, obwohl sie dies zuvor nicht benötigt haben.

Die Frauen spüren, dass sie ihre Beine zunächst weniger einsetzen und kompensieren dies über einen vermehrten Einsatz ihrer Arme.

Eine weitere Erkenntnis der Kinästhetik, die gerade für die Begleitung von PDAFrauen mit eingeschränkter Motorik hilfreich ist, betrifft die Aufgaben unserer Knochen und Muskeln: Wir benutzen unsere Knochen, um unser Gewicht an eine Unterstützungsfläche abzugeben. Unsere Muskulatur ist dann für Bewegung frei. Dies beschreibt das Kinästhetik-Konzept der funktionalen Anatomie im Unterthema Knochen und Muskeln.

\section{Praktische Konsequenzen}

\section{Übertragen auf die PDA bedeutet} dies, dass selbst bei größten motorischen Einschränkungen „nur“ die Muskulatur betroffen ist. Die knöchernen Strukturen sind weiterhin als "Gewichtsschiene“ einsetzbar.

- Im Kreißsaal hat es sich als sehr hilfreich herausgestellt, alle intuitiven
Anpassungen, die die Frau macht, bestärkend zu benennen. Der oben beschriebene vermehrte Einsatz der Arme ist für die Frau manchmal der Schlüssel, ihre jetzt passenden Bewegungswege zu finden und für uns Hebammen ist er ein wichtiger Aspekt, die Frau in ihrem eigenständigen Gebären zu bestärken.

- Die PDA wirkt ca. bis TH 10, das heißt, der obere Brustkorb, die Arme und der Kopf sind für alle Bewegungen uneingeschränkt nutzbar. Auch die Position des Kopfes ist maßgeblich für den Gewichtsverlauf im Körper.

\section{Selbsttest:}

Um sich das bewusst zu machen, können Sie im Stehen den Kopf ein wenig auf eine Seite neigen und dann versuchen, den Fuß auf dieser Seite vom Boden abzuheben.

Sie werden bemerken, dass das nur schwer gelingt, da bei einem geneigten Kopf viel mehr Gewicht auf dieser kompletten Körperhälfte ist.

Manchmal fordert die Geburtsbegleitung mit PDA einen höheren körperlichen Einsatz der Hebamme. Allerdings wird es nie gelingen, die für die Geburt notwendige Bewegung von außen zu machen.

\section{Wir Hebammen sollten uns also} bewusst machen, dass die Frau die Anpassungen in ihrem Körper selbst ausführen muss, d. h. dass es am geburtsförderlichsten ist, wenn sie die weniger einsetzbaren Beine über ihren Oberkörper kompensiert.

Letztlich ist dies ähnlich wie bei Menschen nach einer Querschnittslähmung, denen es auch gelingt, viele Positionswechsel über den Oberkörper selbstständig wieder zu erlernen.

- Beispiel Toilette: In der Praxis könnte also eine mögliche Toilettenvariante sein, dass man eine „Ersatztoilette“ in die Nähe des Kreißbetts baut. Bei eingeschränkter Blasenkontrolle (in der Kinästhetik als ein Phänomen der Haltungsbewegung erklärbar) kann ein Tuch an den Füßen, das die Frau selbst hält, ihr die Möglichkeit geben, das Spannungsnetz in ihrem Körper über die Hände zu steuern, um Wasser lassen zu können (Abb. 1).

- Beispiel: Nach-oben-Rutschen im Bett: Hier ist der Brustkorb am leichtesten beweglich, wenn er möglichst wenig aufliegt. Setzt sich die Frau also zunächst hin, kann sie ihre Arme leichter einsetzen, um sich nach oben zu bewegen. Wenn die Beine zu schwer sind, kann die Hebamme die angestellten Beine halten, damit das Becken leichter beweglich ist. (Abb. 2)

\section{Problem 2: Änderung der Geburtsdynamik}

\section{Die typische Situation}

Alleine die Zeit des Legens der PDA bzw. das Warten auf eine gleichmäßige Ausbreitung der Narkosemittel drosselt erst einmal die Dynamik der Geburt, um die wir uns deshalb nach dem Legen wieder kümmern sollten.

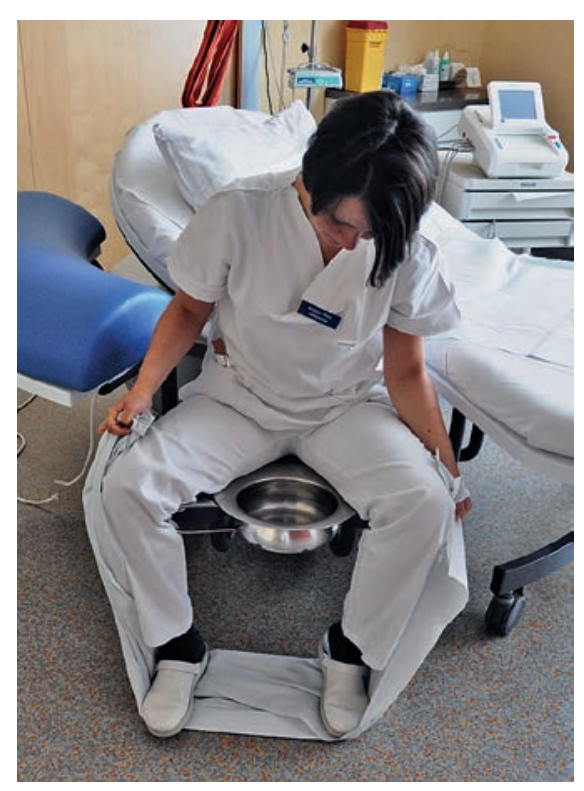

Abb. 1 Ein Steckbecken passt auf den Hocker. Die Möglichkeit zum Ziehen unterstützt die Blasenentleerung. 


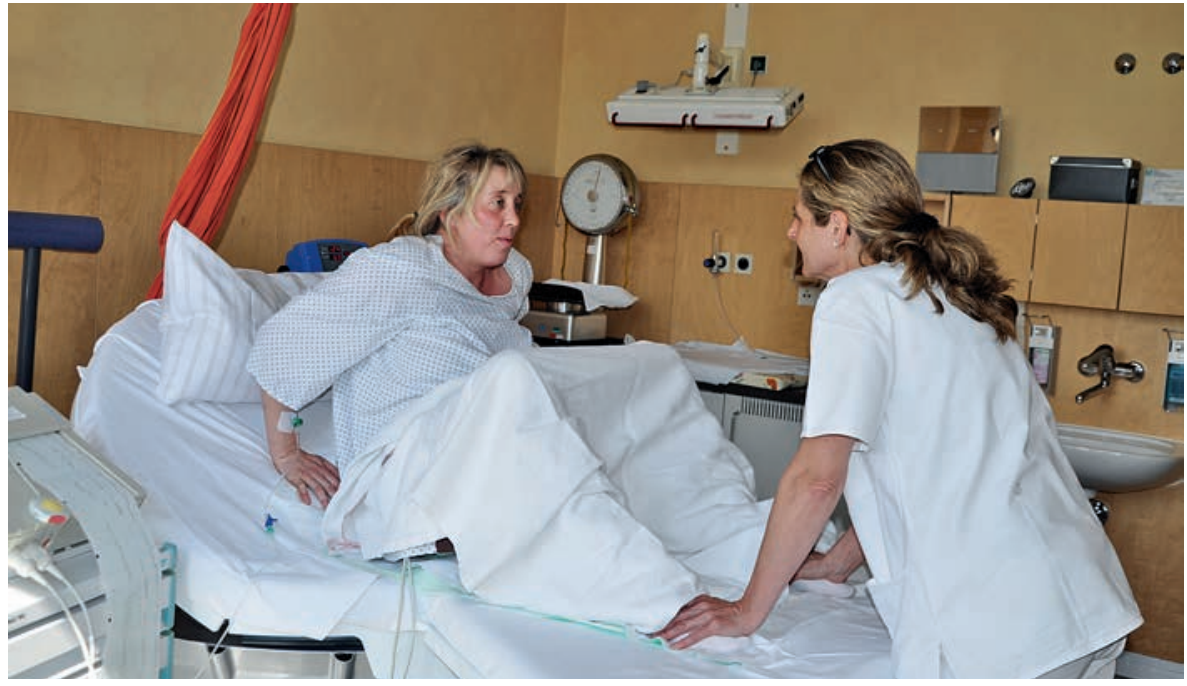

Abb. 2 Das Halten der Füße gibt Stabilität und das Hochrutschen ist für die Frau angenehmer.

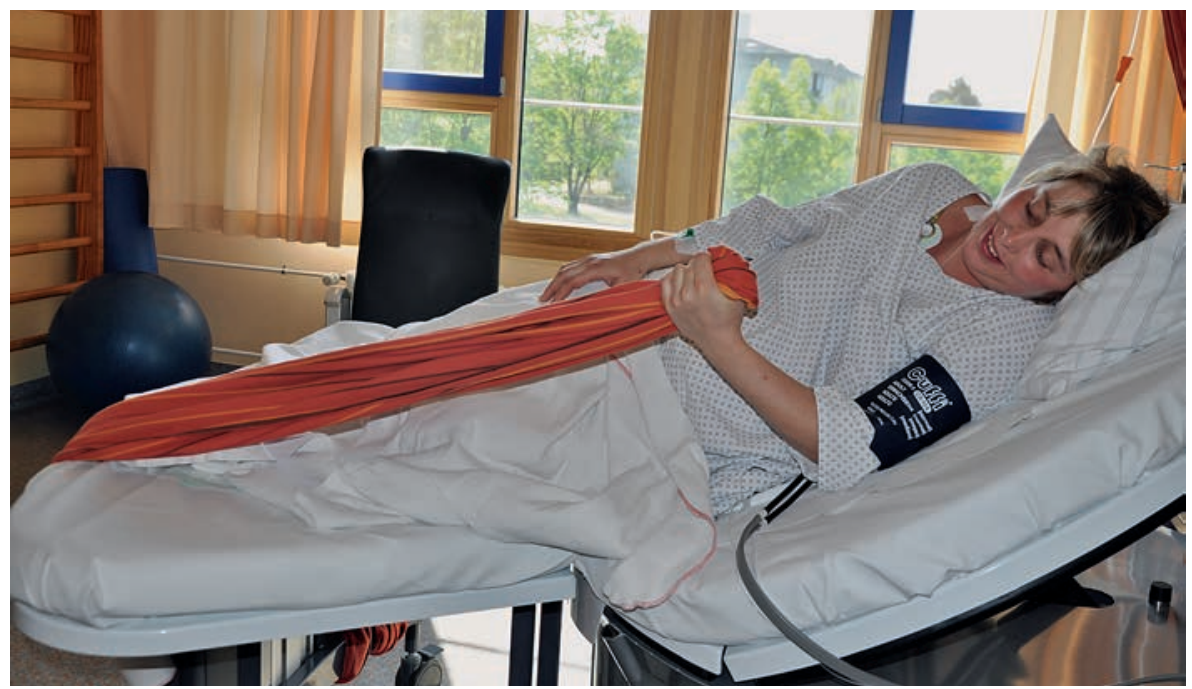

Abb. 3 Der Zug am Tuch lädt das Kind ein, tiefer ins Becken zu rutschen.

\section{Kinästhetische Analyse}

Eine der wichtigsten Grundannahmen in der Kinästhetik ist die Tatsache, dass jeder Mensch nur über die eigene Bewegung sein Leben gestaltet. Sie basiert auf den Forschungen der Verhaltenskybernetik, die den Menschen als geschlossenes System betrachten.

Das bedeutet, dass auch die noch so gut angepassten Angebote der Hebammen erst dann wirken können, wenn sie von der Gebärenden angenommen und selbst umgesetzt werden. len und Kraft tanken können. Sobald die Erholungsphase vorbei ist, sollte man die Frauen körperlich und mental wieder zurück an die Geburtsarbeit führen.

- Eine Möglichkeit, die die Dynamik der Geburt unterstützt, sind Bewegungen, mit der die Frau in jeder Wehe ihr Kind in die Richtung des Geburtswegs einlädt. Dies kann vorsichtig über ein Tuch erfolgen, das um ihre eigenen Füße oder um eine Stange an der unteren Seite des Bettes gebunden ist (Abb.3).

- Sollte die Gebärende mit PDA ihre Wehen nicht mehr spüren, kann der Mann angeleitet werden, die Wehen zu tasten oder mithilfe der Tokometrie seine Frau anzuleiten. Diese Bewegungen unterstützen über den Ferguson-Reflex sanft die Aufrechterhaltung der Wehen.

Gleichzeitig sollte dies ein Signal sein, dass die PDA zu hoch dosiert ist und es einer Anpassung der Medikamentendosis bedarf.

Studien belegen eine große Diskrepanz zwischen der Zufriedenheit der PDAFrauen hinsichtlich der Schmerzausschaltung und der Gesamtzufriedenheit mit der Geburt [1]. Während sie sich häufig sehr zufrieden mit der Schmerzerleichterung äußern, wird die Bewertung des gesamten Geburtsverlaufs wesentlich schlechter eingeschätzt. Die dabei genannte Kritik betrifft retrospektiv die häufigeren Interventionen (vor allem die statistisch belegten höheren Raten an vaginal-operativen Geburtsbeendigungen).

Wir sollten uns also bewusst machen, dass die langfristige Zufriedenheit der Frau sehr mit der aktiven Gestaltung der Geburt korreliert. Deshalb sollten wir unsere persönliche Einstellung zur PDA und die Einschätzung der aktuellen Situation immer wieder überdenken und den Frauen den größten Teil jeder Bewegung selbst zutrauen. 


\section{Problem 3: Aufrechte Gebärhaltungen mit PDA}

\section{Die typische Situation}

Je stärker die Hebamme selbst davon ausgeht, dass bei liegender PDA keine aufrechten Gebärhaltungen möglich sind, desto weniger Chancen wird die Gebärende haben, aufrechte Positionen ausprobieren oder einnehmen zu können.

\section{Kinästhetische Analyse}

Ein genaues Verständnis der Rolle der Knochen und ihrer Anordnung im Körper ermöglicht eine Vielzahl an aufrechten Gebärpositionen (MH-KinaestheticsKonzept der funktionalen Anatomie: Vorder- und Rückseiten).

\section{Beispiel: Wechsel in den Vierfüßlerstand}

Ein Positionswechsel vom aufrechten Liegen zum Vierfüßlerstand ist beispielsweise einfach durchzuführen, wenn die oben beschriebenen Aspekte der Kinästhetik berücksichtigt werden.

- Um den größtmöglichen Bewegungsspielraum des Ober-

körpers zu erreichen, soll die Frau sich zunächst hinsetzen.

- Dreht sie dann den Brustkorb so weit wie möglich zur Seite, verlagert sie ihr Gewicht schon sehr weit in Richtung der unteren Körperhälfte.

- Wenn man das untere Bein (wenn nötig) relativ weit anwinkelt, kann über den Femur (der hier die knöcherne Gewichtsschiene ist), das Gewicht in Richtung Knie fließen (Abb.4).
- Eventuell muss die Frau zunächst einen Schritt nach hinten machen, um den nötigen Spielraum für die Rotation zu haben.

- Dann kann die Hebamme die notwendige Drehung über den Brustkorb unterstützen. Er ist leichter zu bewegen, weil er in dem Moment frei von Gewicht ist und gleichzeitig über die stabile Verbindung durch die Wirbelsäule schnell das Becken mitbewegt.

- Wenn im Kniestand dann die Knie nur geringfügig weiter auseinander sind als hüftbreit, sind die Beine stabil genug.

In der aktiven Geburtsphase sind aufrechte Positionen möglich, wenn wir wieder die knöchernen Strukturen benutzen, um die fehlende Motorik zu kompensieren. 


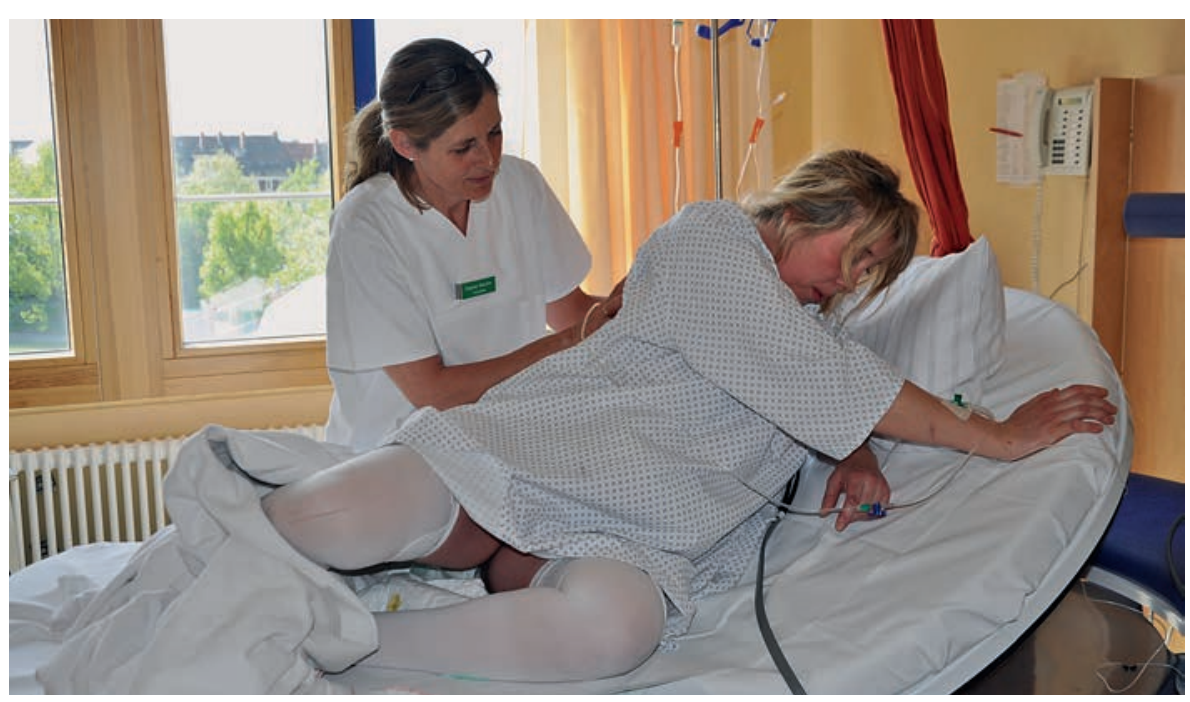

Abb. 4 Die Hebamme unterstützt den Einsatz des Oberkörpers. Das bewirkt eine Drehung des Beckens.

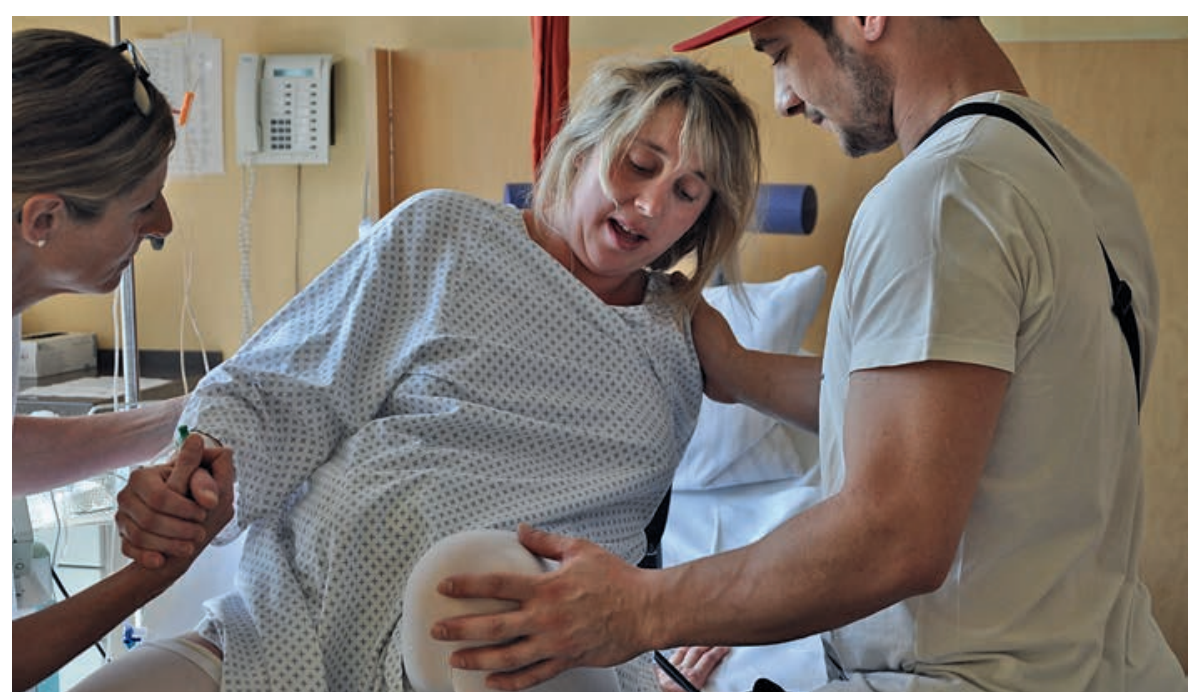

Abb. 5 Bietet man der Frau „nur“ eine Hand an, wird sie die andere hinten abstützen und hat mehr Kontrolle über die Bewegung.

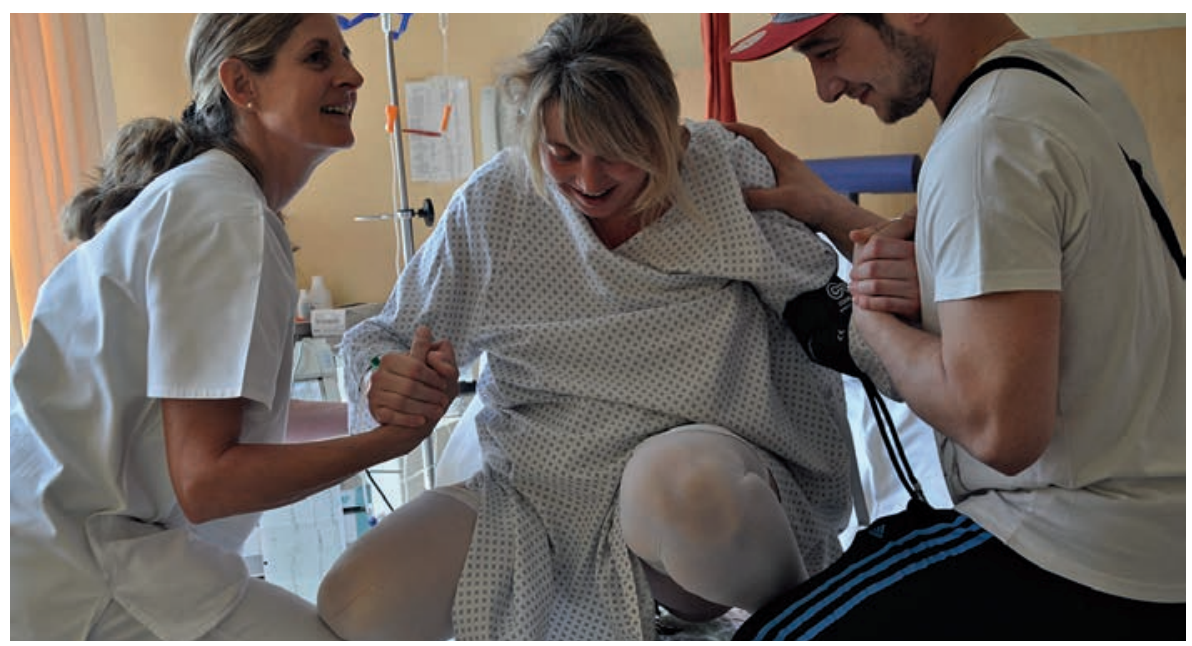

Abb. 6 Die motorisch schwächere rechte Seite wird zuerst abgestützt. Durch das unterstützte Schienbein fühlt sich die Frau stabil und sicher.

\section{Beispiel: Wechsel in die tiefe Hocke}

Startet die Gebärende aus einer sitzenden Position, ist der Weg leichter, als wenn sie noch mit dem Rücken angelehnt ist.

- Über die uneingeschränkt einsetzbaren Arme kann sie ihren Oberkörper nach vorne ziehen (über die Arme ist der Brustkorb leicht beweglich) (Abb.5).

- Die unterstützenden Personen haben ihre freie Hand auf dem Knie der Frau.

- Sobald die Frau beginnt, sich nach vorne zu ziehen, kommt das Becken in Bewegung.

- Über einen Druck auf das Knie kann die Beckenbewegung sehr effektiv unterstützt werden (Abb.6).

- Bei eingeschränkter Motorik kann ein Stillkissen oder der Oberschenkel der beiden Unterstützer das Bein unterstützen. Über das Schienbein erfährt die Frau eine sehr große Stabilität (Abb.6).

Cave: Je höher die Einschränkung der Wahrnehmung der Beine ist, desto wichtiger ist eine sorgfältige Anamnese in Bezug auf Verletzungen des Bewegungsapparats. Wenn eine Vorschädigung der Knie oder Füße besteht, muss unbedingt berücksichtigt werden, dass eine Frau mit PDA die Schmerzen als Schutz vor einer zu weiten Belastung des Gelenkes nicht spürt.

\section{Fazit}

Wir sollten uns bewusst machen, dass die PDA eine Intervention ist, bei der die Frauen nicht weniger, sondern ganz sicher mehr Hebammenbetreuung brauchen, um die beschriebenen Auswirkungen kompensieren zu können.

Aus der täglichen Praxis kennen wir allzu oft die Verläufe, bei der eine Frau mit PDA ihre Hebamme nur noch sieht, wenn es Zeit ist, den Wehentropf zu steigern oder wenn jemand die Muttermunds- 
weite wissen möchte. Und wir wissen, dass diese Geburten oft mit dem Legen der PDA einen Bruch erleben, der schlecht zu kitten ist und dass gerade diese Frauen häufig sectioniert werden müssen.

Die vorgestellten Ideen zeigen Wege, wie die Betreuung der Frauen mit deutlichen Mobilitätseinschränkungen leichter gelingen kann. Als ich vor vielen Jahren die Begründerin der Kinästhetik, Dr. Lenny Maietta, zu aufrechten Gebärpositionen bei einer PDA befragte, war ihre erste Antwort: „Just make it easy“ und wir erarbeiteten gemeinsam einige der hier vorgestellten Ideen. Ich bin sicher, dass es gelingen kann, jede anstrengende Situation stückweise zu vereinfachen. Ein Austausch über bestehende Erfahrungen und individuelle Beobachtungen sind auch hier ein äußerst wichtiger Schritt.

\section{Literatur}

1 Groß M, Dudenhausen JW, Stahl K. Effektive Betreuung während Schwangerschaft und Geburt: Handbuch für Hebammen und Geburtshelfer, Huber 2006

2 Geist C, Harder U, Stiefel A. Hebammenkunde: Lehrbuch für Schwangerschaft, Geburt, Wochenbett und Beruf, Hippokrates 2007

3 Schneider, Husslein, Schneider. Die Geburtshilfe, Springer 2004

4 DHV, Kreißsaaltaschenbuch, Hippokrates 2013

5 Striebel HW. Anästhesie - Intensivmedizin - Notfallmedizin: Für Studium und Ausbildung, Schattauer 2012

6 Institut für Qualität und Wirtschaftlichkeit im Gesundheitswesen, www.iqwig.de

7 www.kinasthetics.com

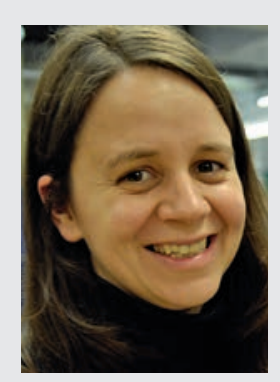

\section{Andrea Mora}

Hebamme im Klinikum Stuttgart und MH Kinaestetics-Trainerin

Libanonstraße 54

70184 Stuttgart

E-Mail: andrea.morapin@gmail.com 